\title{
Levels of Approximation for the Optimal Design of Distributed Energy Systems
}

\author{
Ishanki A. De Mela , Oleksiy V. Klymenko ${ }^{a}$, Michael Short ${ }^{\mathrm{a}^{*}}$ \\ ${ }^{a}$ Department of Chemical and Process Engineering, University of Surrey, Guildford, \\ United Kingdom GU2 7XH \\ *m.short@surrey.ac.uk
}

\begin{abstract}
Optimisation models for the design of distributed energy systems (DES) often exclude inherent nonlinearities and constraints associated with alternating current (AC) power flow and the underlying distribution network. This study aims to assess this gap by comparing the performance of linear and nonlinear formulations of DES design models, connected to and trading with an AC grid. The inclusion of the optimal power flow (OPF) constraints within the DES design framework is demonstrated in the methodology. A residential case study is used to test both models and compare the designs obtained from the two formulations. The results highlight that DES designs obtained are different when constraints related to the underlying distribution network are added, particularly when electricity storage is not considered. Overall, this study highlights the need for future modelling efforts to include OPF within DES optimisation frameworks to obtain practically feasible designs, rather than considering them as standalone problems.
\end{abstract}

Keywords: distributed energy system, optimal power flow, mixed-integer nonlinear programming

\section{Introduction}

The implementation of DES has become increasingly attractive as the power industry aims to integrate more renewable energy resources into existing networks while avoiding costly grid upgrades. DES consist of local, often privately-owned, small-scale energy resources, many of which are renewable. Optimisation models for design and operation of DES are commonly used to determine the suitability and location of the energy resources, and their performance over the system lifetime (Mavromatidis et al., 2019). However, these models often exclude inherent nonlinearities associated with AC power flow, despite most DES generating AC power and interacting with the main electricity grid. Power flow constraints, which are nonlinear and nonconvex, are included in OPF models which determine capacities of distributed generators and calculating active and reactive powers within the network, while maintaining voltage limits (Frank \& Rebennack, 2016). OPF has often been treated as a standalone problem and not adequately accounted for in DES design formulations. Modellers often choose to exclude these nonlinear and nonconvex constraints in DES design models to obtain more computationally tractable models, and achieve an accuracy-complexity balance (De Mel et al., 2020). Mixed-integer linear programming (MILP) models are the most used formulations for DES design, with direct current (DC) approximations that exclude reactive power flows. However, the consequences of doing so have not been adequately analysed, with preliminary studies suggesting that either excluding or linearising certain key constraints can result in practically infeasible designs (Mashayekh et al., 2017; Morvaj et al., 2016). This study addresses the increasing need to compare these different 
levels of approximation and establish which level is most suitable to achieving an accuracy-complexity balance and practically feasible designs.

\section{Methodology}

Four model formulations for DES design are employed in this study: 1) an MILP model with a general active power balance, based on works by Mehleri et al. (2012) for PV constraints and general power balances, and Mariaud et al. (2017) for battery representations and parameters, 2) a nonlinear programming model (labelled NLP-1) serving as a post-optimisation check with fixed binary decisions and capacities obtained from the MILP, and detailed AC power flow constraints, 3) an NLP model similar to model 2 (labelled NLP-2), however with capacities unfixed and treated as variables 4) a mixed-integer nonlinear programming (MINLP) model with detailed AC power flow constraints. All models minimise total annualised cost, which includes technology investment costs, operation and maintenance costs, and income from relevant tariffs. Each of the buildings can install one or more of the following distributed energy resources (DERs) in both models: solar photovoltaics (PVs), lithium-ion batteries, and boilers.

An OPF formulation based on the bus injection model in polar form (Frank \& Rebennack, 2016) for a single-phase, balanced, low voltage network is utilised. It includes nonlinear and nonconvex constraints for net active (Eq. (1)) and reactive power (Eq. (2)), considering node $n \in \mathbf{N}$ and branch $(n, m) \in \mathbf{L}$ :

$$
\begin{aligned}
& P_{n, t}=V_{n, t} \sum_{m=1}^{N} V_{m, t}\left(\left(G_{n m} \cos \left(\theta_{n, t}-\theta_{m, t}\right)+\left(B_{n m} \sin \left(\theta_{n, t}-\theta_{m, t}\right)\right) \quad \forall n \in \mathbf{N}\right.\right. \\
& Q_{n, t}=V_{n, t} \sum_{m=1}^{N} V_{m, t}\left(\left(G_{n m} \sin \left(\theta_{n, t}-\theta_{m, t}\right)-\left(B_{n m} \cos \left(\theta_{n, t}-\theta_{m, t}\right)\right) \quad \forall n \in \mathbf{N}\right.\right.
\end{aligned}
$$

where the variables voltage magnitude and angle are denoted by $V_{n, t}$ and $\theta_{n, t}$, respectively. Note that parameters line conductance $\left(G_{n m}\right)$ and line susceptance $\left(B_{n m}\right)$ are obtained via bus admittance calculations (Frank \& Rebennack, 2016). The OPF formulation also includes inequality constraints to ensure voltage magnitude and angles remain within specified lower and upper bounds. The layout of the network and connections are specified using a bus connectivity matrix for the set of all branches $\mathbf{L}$. Note that a node which injects power has positive power flow, while a node absorbing power has negative power flow. Current limitations are introduced in Eq. (3) where current magnitude in each branch, expressed as a function of voltage, cannot exceed the network-specified maximum current $I^{\max }$ (Frank \& Rebennack, 2016):

$$
\begin{gathered}
\left(V_{n, t} \cos \theta_{n, t}-V_{m, t} \cos \theta_{m, t}\right)^{2}+\left(V_{n, t} \sin \theta_{n, t}-V_{m, t} \sin \theta_{m, t}\right)^{2} \\
\leq \frac{\left(I^{\max }\right)^{2}}{y_{n m}^{2}} \quad \forall(n, m) \in \mathbf{L}
\end{gathered}
$$

where $y_{n m}$ refers to the magnitude of series branch admittance (which is usually calculated in complex form using the parameters series resistance $\left(R_{n m}\right)$ and reactance $\left(X_{n m}\right)$ for each branch). The OPF and MILP are linked by the following equality constraints for each house $i$ connected to node $n$, forming the overall MINLP formulation for DES design (Frank \& Rebennack, 2016):

$$
\begin{aligned}
P_{n, t} & =P_{i, t}^{G e n}-P_{i, t}^{\text {Load }} \\
Q_{n, t} & =Q_{i, t}^{\text {Gen }}-Q_{i, t}^{\text {Load }}
\end{aligned}
$$


These determine whether the respective node is an injection or load node by calculating the net active and reactive powers, respectively. Note that the same OPF formulation is also used in the NLP models, with linear DES constraints and fixed binary decisions.

\section{Results}

\subsection{Case study}

Both models are tested using the residential DES network available in (Morvaj et al., 2016) in a UK context, using seasonal averaged values for electricity demand and heating demand as inputs (note that cooling is not considered in this study). Solar irradiance data obtained from (Met Office, 2020) are also fed as inputs to the model. Demands, available area for PV installation, and available volumes for battery installation at each are summarised in Table 1. Note that the model considers 24 hours (one representative day) for each season, discretised into hourly timesteps. Other input parameters include DER efficiencies, capacity and area of a solar panel, and battery parameters such as maximum state of charge/discharge, volumetric energy density, etc. The DES is assumed to operate under the Feed-In-Tariff scheme (Ofgem, 2019) over 20 years, which provided prosumers with tariffs for renewable energy generation and export. Separate day and night prices are used for buying electricity from the grid to encourage battery installation.

Table 1 . Average daily demands for electricity and heating per day

\begin{tabular}{ccccc}
\hline Building & $\begin{array}{c}\text { Peak Electricity } \\
(\mathrm{kW})\end{array}$ & $\begin{array}{c}\text { Peak Heat } \\
(\mathrm{kW})\end{array}$ & $\begin{array}{c}\text { Area available } \\
\left(\mathrm{m}^{2}\right)\end{array}$ & $\begin{array}{c}\text { Volume available } \\
\left(\mathrm{m}^{3}\right)\end{array}$ \\
\hline A & 3.8 & 10.0 & 150 & 5 \\
$\mathrm{~B}$ & 18.4 & 47.6 & 700 & 5 \\
$\mathrm{C}$ & 14.1 & 27.1 & 600 & 5 \\
$\mathrm{D}$ & 3.8 & 6.4 & 150 & 5 \\
E & 12.0 & 31.3 & 550 & 5 \\
Total & 52.2 & 122.3 & 2150 & 25 \\
\hline
\end{tabular}

The NLPs and MINLP are fed with network-related parameters, such as nominal voltage, resistance $(\mathrm{R})$ and reactance $(\mathrm{X})$, as presented in Figure 1. Note that the power factor $(\mathrm{PF})$ for solar power is set to unity.

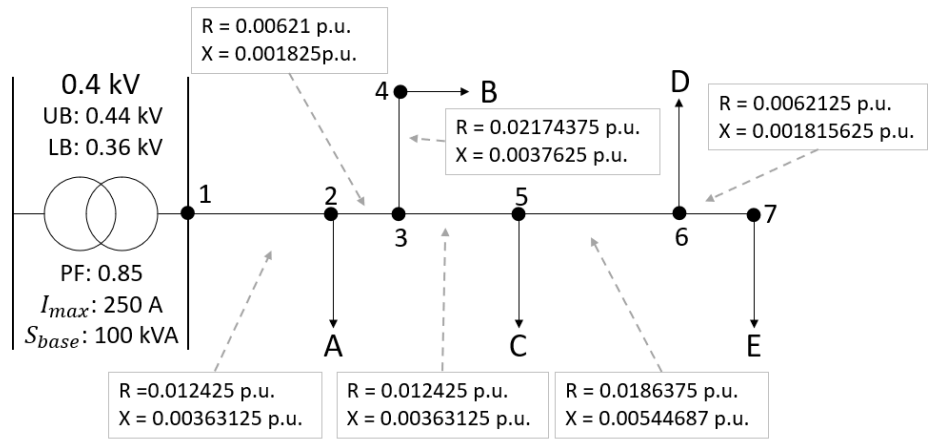

Figure 1. The distribution network and parameters, where $S_{\text {base }}$ is the base apparent power

Two scenarios are tested 1) PVs and boilers only, and 2) PVs, batteries and boilers. 


\subsection{Results and discussion}

The MILP, NLP and MINLP models are solved using CPLEX (IBM, 2019), CONOPT (Drud, 1985), and SBB (Bussieck and Drud, 2001), respectively, all on an Intel ${ }^{\circledR}$ Core $^{\text {TM }}$ i7-10510U processor. It is not possible to guarantee global optimality in the MINLP and NLP solutions, as the formulation is nonconvex and we experienced difficulties using global solvers. The statistics of the models (with all the technologies) are summarised in Table 2. The MINLP and NLPs are initialised using the MILP solution.

Table 2. Number of variables and constraints in the complete models employed in this study.

\begin{tabular}{cccc}
\hline Model & Continuous variables (\#) & Binary variables (\#) & Constraints (\#) \\
\hline MILP & 4,473 & 980 & 9,317 \\
NLP & 8,217 & - & 16,113 \\
MINLP & 8217 & 980 & 16,133 \\
\hline
\end{tabular}

The most notable differences in results were observed in Scenario 1 (PVs and boilers only), which are summarised in Table 3. While the MILP provides the best objective value and chooses the highest total PV capacity of $307 \mathrm{kWp}$, the post-optimisation check conducted via NLP-1 suggests that this solution is unattainable when grid constraints are considered. It is evident that curtailment of solar power is carried out in NLP-1 at nodes 1 and 2 in summer due to current violations in the network, which is also reflected by the lower income and thus an objective value approximately $6 \%$ greater than that of the MILP. This is further confirmed in Figure 2, which shows the current in branch $(1,2)$ (which has the highest current out of all branches) for all four models. Both NLP-2 and MINLP opt to install lower PV capacities of $294 \mathrm{kWp}$ and $287 \mathrm{kWp}$, respectively, to achieve lower objective values than NLP-1, while maintaining grid constraints. The capacities of boilers installed across all four models are the same $(122 \mathrm{kWth})$, which is reflected in the boiler investment and operation costs.

Table 3. Solutions obtained from all four models for the PVs and boilers only scenario.

\begin{tabular}{|c|c|c|c|c|}
\hline Breakdown & MILP & NLP-1 & NLP-2 & MINLP \\
\hline Time taken (s) & 5.29 & 9.07 & 8.82 & 6.21 \\
\hline Objective value (£) & 43,793 & 46,380 & 46,158 & 46,102 \\
\hline Relative optimality gap & 0 & - & - & 0 \\
\hline PV investment $(\mathfrak{E})$ & 54231 & 54231 & 51825 & 50693 \\
\hline Boiler investment (£) & 480 & 480 & 480 & 480 \\
\hline Battery investment $(\mathfrak{£})$ & 0 & 0 & 0 & 0 \\
\hline Grid electricity $(£)$ & 37944 & 37944 & 38294 & 38448 \\
\hline PV operation (£) & 5446 & 5382 & 5160 & 5055 \\
\hline Boiler operation $(£)$ & 5240 & 5240 & 5240 & 5240 \\
\hline Generation income $(\mathfrak{f})$ & 50976 & 48938 & 47310 & 46498 \\
\hline Export income $(\mathfrak{f})$ & 8572 & 7959 & 7532 & 7316 \\
\hline
\end{tabular}




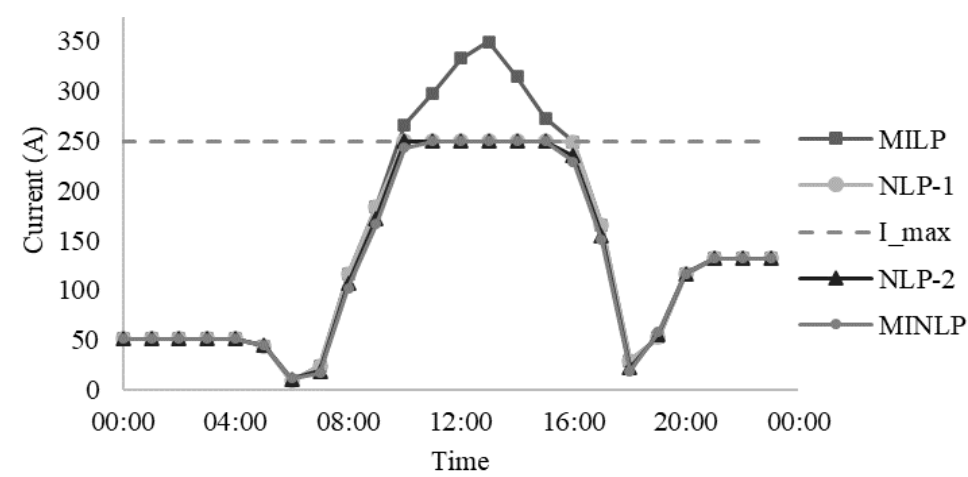

Figure 2. Current in branch $(1,2)$ calculated for all four models. Note that the MILP branch current was calculated via the NLP with current constraints deactivated.

Interestingly, in Scenario 2 where batteries are included in the model formulation, all four models produce the same objective value of $£ 37,566$. The total capacities for PVs (307 $\mathrm{kWp}$ ), batteries (322 kWh), and boilers (122 kWth) across the models are the same. These results suggest that batteries allow the network to compensate for any potential violations by storing power rather than selling all excess power to the grid. Despite the capacities being equivalent, the operational strategies proposed in the models vary, particularly, the times and quantities excess electricity is sold as shown in Figure 3, and/or the batteries are charged. Note that the summation of the magnitudes of these variables across all timesteps and nodes are the same across all four models. In Figure 3, although it appears as if the excess power sold in the nonlinear models are bound at $\sim 100 \mathrm{~kW}$, this is a result of including the current constraint (Eq. (3)) and the value varies between $102.8 \mathrm{~kW}$ and $103.1 \mathrm{~kW}$ in the models. Note that it is necessary to use an OPF formulation to determine the maximum amount of power that can be sold without violating this constraint for each case study, as it is sensitive to the design and parameters used. The MILP operational strategy is tested using a modified version of NLP-1, with fixed variables for selling electricity and charging the battery. This renders the model infeasible, confirming that the MILP strategy is unattainable without violating current constraints.

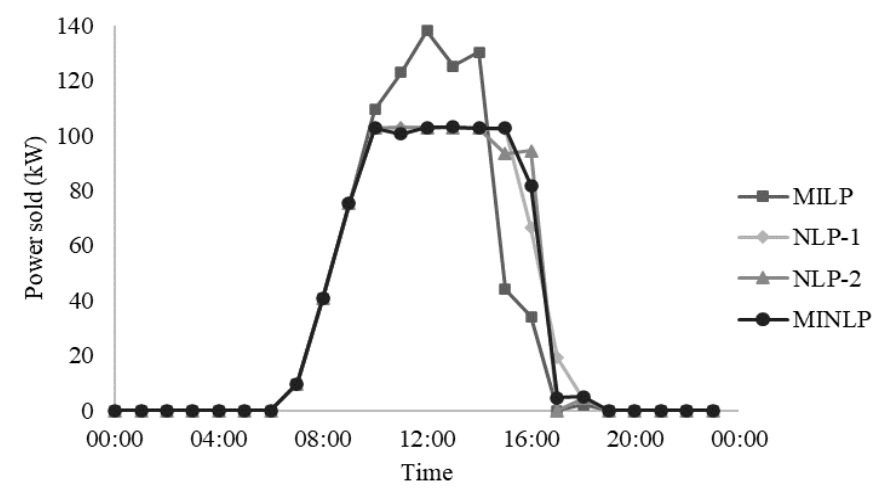

Figure 3. Total excess power sold at each time point.

Despite including nonconvex constraints, the models solved within seconds as highlighted in Table 3 (this included initialisations). However, computational expense may increase significantly if the network is enlarged to include more nodes, particularly if solved as an MINLP. In that case, using a model such as NLP-2, where an MILP is 
solved first to obtain and fix binary decisions, may enable the modeller to achieve an accuracy-complexity balance without excluding network constraints.

\section{Conclusions}

This study focuses on consolidating two previously standalone problems, design of distributed energy systems and optimal power flow, and comparing results from linear and nonlinear model formulations for DES design. The methodology demonstrates the inclusion of nonlinear and nonconvex active and reactive power balances when formulating optimisation models for DES design, as well as other network-related constraints. Results highlight that inclusion of nonconvex constraints guarantees that the design is practically feasible and allows the inclusion of key network constraints that rely on both active and reactive power flow calculations. While the MILP model produces a better objective value when electricity storage is not considered, this is misleading as the inclusion of OPF constraints shows that this would not be realised in practice. Including these constraints also significantly impacts the operation of the DES, regardless of whether electricity storage is available or not, and therefore should be investigated further using DES operational models. Future work includes the extension of the nonlinear model to consider a larger, three-phase unbalanced network where voltage violations may also occur. Other network-related constraints such as line thermal limits and transformer limits will also be considered. Using sensitivity analysis to quantify the effect of uncertainties present in the system and therefore analyse the intermittence of renewable generation may further shed light on the significance of including network constraints.

\section{References}

M.R. Bussieck, A. Drud, A., 2001, SBB: A new solver for Mixed Integer Nonlinear Programming.

I.A. De Mel, O.V. Klymenko, M. Short, M., 2020, Balancing Accuracy and Complexity in Optimisation Models of Distributed Energy Systems and Microgrids: A Review, arXiv.

A. Drud, 1985, CONOPT: A GRG code for large sparse dynamic nonlinear optimization problems, Math. Program., 31, 153-191.

S. Frank, S. Rebennack, 2016, An introduction to optimal power flow: Theory, formulation, and examples, IIE Trans., Institute Ind. Eng., 48, 1172-1197.

IBM, 2019. IMB ILOG CPLEX Optimization Studio V12.9.

S. Mashayekh, M. Stadler, G. Cardoso, M. Heleno, 2017, A mixed integer linear programming approach for optimal DER portfolio, sizing, and placement in multi-energy microgrids, Appl. Energy, 187, 154-168.

G. Mavromatidis, K. Orehounig, L. A. Bollinger, M. Hohmann, J.F. Marquant, S. Miglani, B. Morvaj, P. Murray, C. Waibel, D. Wang, J. Carmeliet, 2019, Ten questions concerning modeling of distributed multi-energy systems, Build. Environ., 165.

E.D. Mehleri, H. Sarimveis, N.C. Markatos, L.G. Papageorgiou, 2012, A mathematical programming approach for optimal design of distributed energy systems at the neighbourhood level, Energy, 44, 96-104.

B. Morvaj, R. Evins, J. Carmeliet, 2016, Optimization framework for distributed energy systems with integrated electrical grid constraints, Appl. Energy, 171, 296-313.

A. Mariaud, S. Acha, N. Ekins-Daukes, N. Shah, C.N. Markides, 2017, Integrated optimisation of photovoltaic and battery storage systems for UK commercial buildings, Appl. Energy, 199, 466-78.

Ofgem, 2019, About the FIT scheme [WWW Document], URL https://www.ofgem.gov.uk/environmental-programmes/fit/about-fit-scheme (accessed 3.18.19). 